Running head: False memories

Can false memories be created through nonconscious processes?

\author{
René Zeelenberg, Gijs Plomp, and Jeroen G. W. Raaijmakers \\ University of Amsterdam
}

Address for correspondence:

René Zeelenberg

Psychology Department

Indiana University

Bloomington, IN 47405

USA

phone: (812) 855-0626

fax: (812) 856-5217

email: rzeelenb@indiana.edu 


\begin{abstract}
Presentation times of study words presented in the Deese/Roediger and McDermott (DRM) paradigm varied from $20 \mathrm{~ms}$ to $2000 \mathrm{~ms}$ per word in an attempt to replicate the false memory effect following extremely short presentations reported by J.G. Seamon, C.R. Luo and D.A. Gallo (1998). Both in a within-subjects design (Experiment 1) and in a between-subjects design (Experiment 2) subjects showed memory for studied words as well as a false memory effect for related critical lures in the 2000-ms condition. However, in the conditions with shorter presentation times (20 ms in Experiment 1; $20 \mathrm{~ms}$ and $40 \mathrm{~ms}$ in Experiment 2) no memory for studied words, nor a false memory effect was found. We argue that these experiments do not support the claim for a nonconscious basis of the false memory effect.
\end{abstract}




\section{Can false memories be created through nonconscious processes?}

How illusive are our memories? Can we learn things that we do not consciously perceive? Although we can in general be reasonably sure that our memories of events are quite accurate, recent research seems to indicate that under certain circumstances we can not be entirely sure about what we think we remember (Roediger \& McDermott, 1995) and that learning can occur under conditions where words are almost invisible (Seamon, Luo, \& Gallo, 1998). Deese (1959) has shown that it is possible to create illusory memories for words that subjects did not previously study. Deese's subjects studied lists of words (e.g., thread, pin, eye, etc.) that were semantically associated to a nonpresented word, the critical lure (e.g., needle). On an immediate free recall test, subjects tended to reproduce not only the studied list items but also the nonstudied critical lure. Roediger and McDermott (1995) have brought these false memories again to our attention by replicating and extending Deese's original findings. They found that in a recognition test, subjects indicated to recognize nonstudied critical lures at a rate that was comparable to the hit rate for studied list items. Together these findings constitute a powerful illusion of memory.

Elaborating on the Roediger and McDermott (1995) findings, Seamon et al. (1998) reported false recognition of critical lures even when study items were presented for extremely short presentation times. In these experiments, subjects studied lists for a presentation time ranging from $20 \mathrm{~ms}$ to $2000 \mathrm{~ms}$ per word. On a subsequent recognition memory test, subjects indicated whether a presented word was previously studied or not and displayed false memories for critical lures in all presentation time conditions. In the 20-ms condition, a false memory effect was obtained even for subjects who appeared not to be able to discriminate studied list items from distractors. These results were obtained in both a between-subjects and a withinsubject design. In other studies Seamon and colleagues (Seamon, Luo, Schlegel, Greene, \& Goldberg, 2000; Seamon, Luo, Schwartz, Jones, Lee, \& Jones, 2002) have replicated the finding of a false recognition effect in the 20-ms condition. It seems that we must draw the quite amazing conclusion that we do not only 'remember' words that were never presented, but also 
that we 'remember' nonpresented words without any memory for the words that give rise to this 'memory'.

Seamon et al. (1998) explained their results by assuming that the false memory effect can occur as a result of conscious as well as nonconscious processes. A key role in their explanation is the notion of implicit activation. On this account, the presentation of multiple associated words can result in the repeated conscious or nonconscious activation of associatively related words. Such indirectly activated words could later be perceived as genuine memories, due to their relatively strong level of activation. Seamon et al. argued that their results indicate that false memories can be based on nonconscious activation of semantic concepts during list presentations.

The finding of false memories at a presentation time of only $20 \mathrm{~ms}$ is quite surprising. At a speed of $20 \mathrm{~ms}$ per word subjects are generally not able to identify the words they are being shown on the display. As no conscious attention can be given to these words, memory for them should be very poor or absent (e.g., Fisk \& Schneider, 1984; Crabb \& Dark, 1999). That the nonconscious activation of words that are associatively related to these very shortly presented stimuli can yield long-term memories seems at odds with theories of memory that generally assume that long-term memory depends on conscious attention (Craik \& Tulving, 1975; Raaijmakers \& Shiffrin, 1981; Shiffrin \& Schneider, 1977).

In our view, however, the conclusion that nonconscious processes are (partially) responsible for the false memory effect is premature and cannot be drawn from the results reported by Seamon et al. $(1998,2000,2002)$. One problem with this conclusion is that subjects were still able to discriminate studied items from nonstudied items in the 20-ms condition. Seamon et al. (1998) observed hit rates for studied list items in the different 20-ms conditions that were consistently higher than the false alarms rates for nonstudied list items. Depending on the memory load condition, this difference varied from $8 \%$ (memory load present, Experiment 1 ) to $19 \%$ (memory load absent, Experiment 1) in the Seamon et al. (1998) study. Using a 20-ms condition with no memory load and lists of category exemplars, the difference between hit rate 
and false alarm rate in the Seamon et al. (2000) study was even higher and varied from $24 \%$ (Experiment 2B) to 30\% (Experiment 2A). For words presented for only $20 \mathrm{~ms}$, differences between hit rate and false alarm rate of this size are quite surprising, to say the least. Because subjects could apparently identify some of the list items and store them in memory the conclusion that false memories are based on nonconscious processes is problematic.

In the present study we attempted to replicate the finding of a false memory effect following briefly presented lists of words using a experimental set-up that was specifically designed to present words for very short presentation times. We expected to find a false memory effect in the conditions in which subjects studied the words for as long as $2000 \mathrm{~ms}$. The question of interest, however, was if we would be able to replicate the finding of false memories in the 20-ms condition.

\section{Experiment 1}

Memory for list items and critical lures was tested for short $(20 \mathrm{~ms})$ and long $(2000 \mathrm{~ms})$ presentation times in a within-subjects design that was adapted from Experiment 2 of Seamon et al. (1998). As we were primarily interested in replicating the false memory effect for briefly presented lists of words, our experiment differed from that of Seamon et al. in that memory load as an experimental variable was omitted from the design.

\section{Method}

Subjects. The subjects were 32 students of the University of Amsterdam who received course credit for their participation. All participants were native speakers of Dutch and reported normal or corrected-to-normal vision. Care was taken to ensure that none of them had previously participated in a related memory experiment.

Materials and Apparatus. A subset of the lists used by Zeelenberg and Pecher (2002) were used in the present experiment. These lists were originally normed in English by Stadler, Roediger, \& McDermott (1999) and translated into Dutch by Zeelenberg and Pecher. Each list was centered around one critical lure. For example, for the critical lure naald (English translation equivalent: needle) the list items consisted of the following words: draad (thread), oog (eye), 
speld (pin), garen (thread), naaien (sewing), pijn (pain), acupunctuur (acupuncture), scherp (sharp), punt (point), vingerhoedje (thimble), prik (prick), injectie (injection). From the original 36 lists normed by Stadler et al. (1999) 16 were selected and divided into four sets of four lists for the purpose of counterbalancing across the experimental conditions. Each of the 16 lists consisted of 12 items and one critical lure. List items were placed in order of associative strength to the critical lure using the de Groot (1980) free association norms, such that the words that were most strongly related to the critical lure were on top of the list and words that were least strongly associated with the critical lure were positioned at the end of each list. For words not listed in the de Groot norms, the position in the list was based on the authors' judgment of the associative strength between the list item and critical lure.

In order to ensure the accuracy of the short presentation times in our experiment, a Hewlett Packard digital display module, model HP1345A, was used. The HP1345A display is a point-plot or vector-type display rather than the more common raster-type displays such as used in desktop PC's. The advantage of such a display is that it is not limited by a particular refresh rate. That is, with a normal display the beam will always move at a fixed rate (determined by the refresh rate) from the top left corner to the bottom right corner of the screen. As a result, the presentation time can only be varied in multiples of the refresh time (i.e., in steps of $20 \mathrm{~ms}$ with a $50 \mathrm{~Hz}$ display or $13.9 \mathrm{~ms}$ with a $72 \mathrm{~Hz}$ display). A point-plot display such as the one used in the present experiments does not have that limitation but allows direct control of the position of the beamer. As a result, when only a few characters (such as a word) are to be displayed it will take much less time to write those characters (usually about $1 \mathrm{~ms}$ or so). The software that was used to program the display module allows variation of the presentation time in steps of $2 \mathrm{~ms}$ and has been used previously in studies investigating masked semantic priming (Pecher, Zeelenberg, \& Raaijmakers, 2002) and repetition priming in perceptual identification (Wagenmakers, Zeelenberg, \& Raaijmakers, 2000). The display module and the response collection were controlled by an IBM Personal Computer running under DOS. 
Procedure. Our procedure closely followed that of Seamon et al. (1998). Subjects were told that they were participating in an experiment that was about memory and were instructed to look attentively at the screen in front of them in order to memorize as many words as possible for a recognition test that would follow at the end of the experiment. No mention was made of the associative relatedness of the words within each list. In order to familiarize the participants with the experimental procedure, two lists were presented as practice lists. One of these was presented at a speed of $20 \mathrm{~ms}$ per word, the other at $2000 \mathrm{~ms}$ per word.

The eight lists were presented in two blocks of four lists, with a short break after the fourth list. Within each block, two successive lists were presented at a rate of $2000 \mathrm{~ms}$ per word and two lists were presented at a rate of $20 \mathrm{~ms}$ per word. For half of the subjects both blocks began with two lists presented in the 2000-ms condition and ended with two lists presented in the 20-ms condition, for the other half of the subjects this order was reversed.

Words were presented in capitals and appeared centered on the screen that was located approximately $50 \mathrm{~cm}$ in front of the subject. The critical lures were, of course, never presented. Each word on the list was presented immediately following the preceding word (i.e., the ISI was $0 \mathrm{~ms}$ ) and each list concluded with the words 'NIEUWE LIJST' (Dutch equivalent of 'NEXT LIST') presented immediately following the last word on the list. The words 'NIEUWE LIJST' remained on the screen for $1 \mathrm{~s}$. Immediately after the fourth list and after the final list the word 'EINDE' (Dutch equivalent of 'END') was presented (instead of 'NEXT LIST'). All stimuli were presented on the same location on the screen.

After the eight lists were studied, subjects were given a recognition test in which they had to judge whether or not a test word had occurred on one of the study lists. All items again appeared capitalized and centered on the screen. The test words consisted of the words with position numbers 1,8 and 10 on each of the 16 study lists that were used in the experiment, as well as the critical lures belonging to these lists. Thus, of the total of 64 words presented at test, 24 words had been studied in either the 20 -ms or 2000 -ms condition, 8 words were the critical lures belonging to the 8 studied lists and the remaining 32 words belonged to the lists that were 
not studied (24 list items and 8 critical lures). If subjects thought they recognized a presented word as a studied item they were instructed to press the /?-key of the keyboard ('old' response) and if they did not recognize the word as a list item they were instructed to press the z-key ('new' response). All items in the recognition test were presented in a different random order for each subject and remained on the screen until a response was made.

\section{Results and Discussion}

The results of Experiment 1 are summarized in Table 1, which shows the percentage of 'old' responses as a function of study condition. A clear false memory effect was obtained in the 2000-ms condition. Subjects gave an 'old' response to $86.7 \%$ of the critical lures from studied lists and to only $7.8 \%$ of the critical lures from nonstudied lists. This false memory effect was highly significant, $t(31)=17.38, p<.0001$. Subjects correctly recognized $87.0 \%$ of the list items from studied lists, while the false alarm rate for nonstudied list items was $4.2 \%$. This difference was also significant, $t(31)=34.59, p<.0001$. In the $20-\mathrm{ms}$ condition, however, only $7.0 \%$ of the list items and $9.4 \%$ of the critical lures from studied lists received an 'old' response. This is almost identical to the overall false alarm rates for nonstudied list items, and the false alarm rate for critical lures belonging to nonstudied lists. T-tests revealed that neither the false memory effect, nor the veridical memory effect were significant, $t(31)=0.61, p>.25$ and $t(31)=1.53, p$ $>.10$, respectively. Thus, in the 20 -ms condition there was no evidence for memory for the presented words as well as no evidence for false recognition of the critical lures.

Although a clear false memory effect was found in the 2000-ms condition no false memory effect was found in the 20 -ms condition. We were thus unable to replicate the crucial result of the Seamon et al. (1998) study. One possible reason for our failure to obtain evidence for false recognition in the 20 -ms condition might be that a high criterion for recognition was used by the subjects in our experiment. Overall false alarm rates for list items were rather low in the present experiment. Our subjects were quite accurate in discriminating list items presented for $2000 \mathrm{~ms}$ from nonstudied list items. Therefore, it is possible that they used a high criterion for giving an 'old' response. As a result the studied list items as well as the critical lures in the 
20-ms condition might not have been familiar enough to pass the criterion for an 'old' response. Another explanation for the present results is of course that no long-term memory traces were formed in the 20-ms condition, because subjects could not consciously identify the items on the study lists. We decided to explore these issues further in another experiment.

Experiment 2

In our next attempt to replicate the false memory effect under extremely short presentation times we used a between-subjects design similar to Seamon et al. (1998, Exp. 1), again not manipulating memory load. Furthermore, we chose to employ a 40-ms condition (instead of the 250-ms condition used by Seamon et al.) as our first experiment clearly showed a false memory effect with longer presentation times and because the replication of the false memory effect with extremely short presentation times was our main goal.

The rationale for using a between-subjects design was that if subjects are confronted with only one experimental condition they might be more inclined to use a low criterion for recognition for very briefly presented words. For the conditions with extremely short presentation times we expected more false alarms to be made, leaving more room for false memories to become manifest.

\section{Method}

Subjects. The participants were 48 students of the University of Amsterdam who received course credit for their participation. None of the subjects had participated in Experiment 1, or any other related memory experiment. All subjects were again native speakers of Dutch and reported normal or corrected-to-normal vision. Sixteen subjects each were randomly assigned to one of the three presentation time conditions (20 ms, $40 \mathrm{~ms}$, or $2000 \mathrm{~ms}$ ).

Materials, design and procedure. Each subject viewed two practice lists to become familiar with the presentation procedure in the experiment. The presentation time of the practice lists depended on the experimental condition to which the subject was assigned and was identical to that in the experiment proper. For counterbalancing purposes the word lists were divided into two sets of 8 lists, set A and set B. Half of the subjects studied set A, the other half studied set B. 
After the presentation of the list items, subjects were given the same recognition test as in Experiment 1 . In the $20-\mathrm{ms}$ and in the $40-\mathrm{ms}$ conditions subjects were encouraged to 'follow your intuition' and to 'guess if you really don't know' in an attempt to lower their criteria for responding 'old'. All other aspects of the method were identical to those of Experiment 1.

\section{Results and Discussion}

Table 2 summarizes the results of Experiment 2, displaying the percentage of 'old' responses on the recognition test as a function of study condition. In the 2000 -ms condition, an 'old' response was given to $77.1 \%$ of the list items and $73.4 \%$ of the critical lures from studied lists. The number of false alarms to list items and critical lures from nonstudied lists were low. For lists items, the percentage of 'old' responses was significantly higher for studied lists than for nonstudied lists, $t(15)=27.45, p<.0001$, showing veridical memory. For critical lures also, the percentage of 'old' responses was significantly higher for studied lists than for nonstudied lists, $t(15)=11.42, p<.0001$. These results replicate the false memory effect as it was previously found in Experiment 1, and by Seamon et al. (1998) and others.

In contrast, there was again no evidence for false memories in the 20-ms and 40-ms conditions. Statistical tests showed that the differences in the percentage of 'old' responses to critical lures for studied and nonstudied lists were not significant, $t(15)=0.10, p>.25$ and $t(15)$ $=0.32, p>.25$, for the $20-\mathrm{ms}$ condition and $40-\mathrm{ms}$ condition, respectively. Veridical memories were also absent for the short presentation conditions as the differences in the percentage of 'old' responses to list items from studied and nonstudied lists were not significant, $t(15)=0.70, p>$ .25 and $t(15)=0.73, p>.25$, for the 20 -ms condition and $40-\mathrm{ms}$ condition, respectively.

The results of Experiment 2 closely resemble those of Experiment 1. We again found a strong false memory effect in the 2000-ms condition but observed no such effect in the conditions with a shorter presentation time. Despite the fact that subjects in the 20-ms and 40-ms condition of Experiment 2 tended to give relatively more 'old' responses than subjects in the 20ms condition of Experiment 1, they did not display any memory for studied list items. Neither 
did they show a false memory effect for critical lures as their number of false alarms did not differ between studied and nonstudied lists.

\section{General Discussion}

In two experiments aimed at replicating the false memory effect for lists of words that were presented for extremely short presentation times at study, we have obtained no evidence for false memories in these conditions. This finding was obtained in both a within- and a betweensubjects design. The absence of a false memory effect was obtained even for the short presentation condition in which we increased the presentation time to $40 \mathrm{~ms}$ instead of $20 \mathrm{~ms}$. Also important is the finding that we did not obtain any evidence for veridical memories in these conditions. In the 2000-ms condition, false memories occurred for the critical lures belonging to the lists presented at study. Moreover, false memories occurred at a rate that was nearly comparable to the recognition of studied words. All in all we have successfully replicated the false memory effect in the 2000-ms conditions, but have been unable to find such an effect in conditions with an extremely short presentation time of list items.

To address possible concerns about the power of the present experiments to detect a false memory effect for the short presentation conditions we performed power analyses. Our estimate of the effect size was based on the results reported by Seamon et al. (1998, 2002, 2002). Using G*Power (Buchner, Faul, \& Erdfelder, 1997), we found that the power to detect a false memory effect (defined as the difference in the percentage of 'old' responses to the critical lures from studied lists and that from nonstudied lists) of 15\% was .99 (one-tailed) in Experiment 1. To calculate the power of Experiment 2 we collapsed the results from the 20-ms and 40-ms conditions because the results for these conditions were almost identical. These collapsed data showed a $-1 \%$ false memory effect, which was, of course, not significant, $t(31)=0.24, p>.25$. The power to detect a false memory effect of $15 \%$ was .91 (one-tailed). We conclude that is highly unlikely that the absence of a false memory effect in our study was due to a lack of power to detect such an effect. 
Although the results confirmed our expectations, it remains unclear exactly why Seamon et al. $(1998,2000)$ were able to consistently find a false memory effect in the 20 -ms condition whereas we did not find such a result. The results do not seem to depend on the particular materials used in the Seamon et al. (1998) study as Seamon et al. (2000) replicated the false memory effect using different materials. One notable difference between our results and those of Seamon et al. $(1998,2000)$ is that in the Seamon et al. studies, subjects performed above chance for list items even in the 20-ms condition, whereas we obtained no evidence for veridical memories at short presentation times. Surprisingly, Seamon et al. observed that hit rates for studied items in the different 20-ms conditions were consistently higher than false alarms rates for nonstudied items (in some cases by as much as 20-30\%). One admittedly speculative but not unreasonable explanation is that in the Seamon et al. studies due to the combination of software and hardware used words were (sometimes) being presented for more than $20 \mathrm{~ms}$ and that as a result subjects could occasionally identify the list items. Seamon et al. (1998) give no information about the hardware and software that were used other than that the words were presented on an Apple computer. However, presenting a sequence of 15 words for precisely 20 ms each is difficult to accomplish using standard software and equipment and would require special precautions. This is especially the case when experiments are run on PC's running under modern operating systems such as Windows or the Macintosh operating system. Under these operating systems the actual durations may deviate considerably from the intended durations (see Schneider, Eschman \& Zuccolotto, 2002, p. 76-77). In view of the fact that in the experiments of Seamon et al. recognition performance was still surprisingly good in the 20 -ms condition, the claim that the presentation times were indeed $20 \mathrm{~ms}$ should perhaps not be taken at face value.

Whether this explanation is correct or not, the mere fact that Seamon et al. obtained false memories in the 20-ms condition does not support the claim that the false memory effect was due to nonconscious processes, because even at these extremely short presentation times memory for list items was above chance. Seamon et al. did not ask subjects to report whether they could identify any words in the 20-ms condition. Thus, we have no way of knowing for sure whether or 
not subjects could occasionally identify one or more words. Their results strongly suggest, however, that this was the case (unless one would want to argue that veridical memory too was based on nonconscious processes). If subjects could indeed identify some words, the claim for a nonconscious basis of false memories would of course be much less convincing.

In an attempt to alleviate some of the problems in the interpretation of their results, Seamon et al. (1998) performed a median-split analysis on their results creating two groups of subjects: so-called bad memorizers and good memorizers. This analysis showed that a false memory effect was present for the bad memorizers although they performed at chance level in their recognition of list items. On the basis of these results Seamon et al. claimed that false memories were due to nonconscious activation. One problem with this argument is that it is based on the assumption that the median-split analysis provides an unbiased and accurate estimate of veridical and false memories. Selecting subjects on the basis of their observed performance is somewhat problematic when those observed scores are subject to random error. This is especially a problem when the score on which the median-split is made is a difference score (hit rate minus false alarm rate) since such difference scores are known to be less reliable (more variable) than the original scores. Since no indication was given about the reliability of these differences, it remains unclear to what extent the selected groups of good and bad memorizers reflect groups that truly differ in memory accuracy. To the extent that they do not, it is not surprising that there is no relation between performance on the list items versus the critical lures. Thus, without further information, the median-split analysis does not unambiguously support the claim that false memories arise from nonconscious processes. Another problem with this analysis is that selecting subjects out of a counterbalanced design might destroy the design because items are no longer equally often presented in the studied and nonstudied conditions (see McKoon \& Ratcliff, 1996, for a more elaborate discussion of this problem). Seamon et al. did not report if their counterbalanced design was still intact for the so-called bad memorizers.

Finally, we mention that Seamon et al. $(1998,2000,2002)$ are not the only ones who have reported false memories for lists of words that were presented for very short durations. A 
recent study by McDermott and Watson (2001; see also Roediger, Robinson, \& Balota, 2001, cited in Roediger, Balota, \& Watson, 2002) included what they called a 20-ms condition. However, McDermott and Watson acknowledged that due to their monitor refresh rates the actual presentation rate in their study approximated $32 \mathrm{~ms}$. Maybe more important, the presentation procedure included a 32-ms ISI between subsequent presentations of the list items. Hence, the total time between the presentation of subsequent items was approximately 64 ms. Under these conditions it is conceivable that subjects were able to identify some of the items on the study list. McDermott and Watson did not report whether subjects were able to consciously identify some of the list items. However, given that subjects recalled about $80 \%$ of the words presented on the last position of the study list, it seems very unlikely that these words had not been consciously identified by the subjects. Therefore, these studies provide no evidence that false memories are due to nonconscious processes (nor was this the aim of these studies). To be clear, our claim is not that false memories will be absent whenever presentation times are short. At some presentation duration subjects will be able to identify some of the words on the study list and these words may give rise to false memories. However, this provides no evidence for a nonconscious basis of false memories.

To summarize, the results observed in the 2000-ms condition of both our experiments is a clear indication that our stimulus lists were adequate in generating false memories. The false memory effect for nonstudied critical lures that are strong semantic associates of words studied in lists is a robust experimental effect that can be easily demonstrated when the list items are presented for a presentation time at which they can be easily identified. The story is completely different, however, when the list items are presented for such a short time that they cannot be identified. As far as we are concerned, the false memory effect does not hold under such conditions. Accordingly, caution should be taken when inferring that false memories arise from nonconscious processes. 


\section{References}

Buchner, A., Faul, F., \& Erdfelder, E. (1997). G*Power: A priori, post-hoc, and compromise power analyses for the Macintosh (Version 2.1.2) [Computer program]. Trier, Germany: University of Trier.

Crabb, B. T., \& Dark, V. J. (1999). Perceptual implicit memory requires attentional encoding. Memory \& Cognition, 27, 267-275.

Craik, F. I. M., \& Tulving, E. (1975). Depth of processing and the retention of words in episodic memory. Journal of Experimental Psychology: General, 104, 268-294.

Deese. J. (1959). On the prediction of the occurrence of particular verbal intrusions of words in immediate recall. Journal of Experimental Psychology, 58, 17-22.

Fisk, D., \& Schneider W. (1984). Memory as a function of attention, level of processing, and automatization. Journal of Experimental Psychology: Learning, Memory, and Cognition, 10, $181-197$

de Groot, A. M. B. (1980). Mondelinge woordassociatienormen: 100 woordassociaties op 460 Nederlandse zelfstandige naamwoorden. [Oral word association norms: 100 word associations to 460 Dutch nouns]. Lisse, The Netherlands: Swets \& Zeitlinger.

McDermott, K. B., \& Watson, J. M. (2001). The rise and fall of false recall: The impact of presentation duration. Journal of Memory and Language , 45, 160-176.

McKoon, G., \& Ratcliff, R. (1996). Separating implicit from explicit retrieval processes in perceptual identification. Consciousness and Cognition, 5, 500-511.

Pecher, D., Zeelenberg, R., \& Raaijmakers, J.G.W. (2002). Associative priming in a masked perceptual identification task: Evidence for automatic processes. Quarterly Journal of Experimental Psychology, 55A, 1157-1173.

Raaijmakers, J. G. W., \& Shiffrin, R. M. (1981). Search of associative memory. Psychological Review, 88, 93-134.

Roediger, H. L., Balota, D. A., \& Watson, J. M. (2002). Spreading activation and arousal of false memories. In H. L. Roediger, J. S. Nairne, I. Neath, \& A. M. Surprenant (Eds.), The 
nature of remembering: Essays in the honor of Robert G. Crowder (pp. 95-115). Washington, DC: American Psychological Association.

Roediger, H. L., \& McDermott, K. B. (1995). Creating false memories: Remembering words not presented in lists. Journal of Experimental Psychology: Learning, Memory and Cognition, 21, 803-814.

Seamon, J. G., Luo, C. R., \& Gallo, D. A. (1998). Creating false memories of words with or without recognition of list items: Evidence for nonconscious processes. Psychological Science, 9, 20-26.

Seamon, J. G., Luo, C. R., Schlegel, S. E., Greene, S. E., \& Goldenberg, A. B. (2000). False memories for categorized pictures and words: The category associates procedure for studying memory errors in children and adults. Journal of Memory and Language, 42, 120-146.

Seamon, J. G., Luo, C. R., Schwartz, M. A., Jones, K. J., Lee, D. M., \& Jones, S. J. (2002). Repetition can have similar or different effects on accurate and false recognition. Journal of Memory and Language, 46, 323-340.

Schneider, W., Eschman, A., \& Zuccolotto, A. (2002). E-Prime User's Guide. Pittsburgh: Psychology Software Tools Inc.

Shiffrin, R. M., \& Schneider, W. (1977). Controlled and automatic human information processing II: Perceptual learning, automatic encoding, and a general theory Psychological Review, 84, 127-190.

Stadler, M. A., Roediger, H. L., \& McDermott, K. B. (1999). Norms for word lists that create false memories. Memory \& Cognition, 27, 494-500.

Wagenmakers, E.M., Zeelenberg, R., \& Raaijmakers, J.G.W. (2000). Testing the counter model for perceptual identification: Effects of repetition priming and word frequency. Psychonomic Bulletin \& Review, 7, 662-667.

Zeelenberg, R., \& Pecher., D. (2002). False memories and lexical decision: Even twelve primes do not cause long-term semantic priming. Acta Psychologica, 109, 269-284. 


\section{Author Note}

René Zeelenberg was supported by a grant from the Foundation for Behavioral and Social Sciences of the Netherlands Organization for Scientific Research. We thank Mirte Bakker for her help in constructing the stimulus materials and Mark Rotteveel for interesting discussions. Mike Masson, Roddy Roediger and John Seamon provided helpful comments on an earlier version of the manuscript.

Correspondence concerning this article can be addressed to René Zeelenberg, Psychology Department, Indiana University, Bloomington, IN 47405. E-mail may be sent to rzeelenb@indiana.edu. 
Table 1

Percentage of 'Old' Responses in Experiment 1 as a Function of Study Condition

Study Condition $\quad \%$ Old

Studied List

$2000 \mathrm{~ms}$

List Item

87.0

Critical Lure

86.7

$20 \mathrm{~ms}$

List Item $\quad 7.0$

Critical Lure $\quad 9.4$

Nonstudied List

$\begin{array}{ll}\text { List Item } & 4.2 \\ \text { Critical Lure } & 7.8\end{array}$

Note. Presentation time was varied within subjects. 
Table 2

Percentage of 'Old' Responses in Experiment 2 as a Function of Study Condition

Condition Studied List Nonstudied List

$2000 \mathrm{~ms}$

List Item $\quad 77.1 \quad 5.0$

Critical Lure $\quad 73.4 \quad 10.9$

$40 \mathrm{~ms}$

$\begin{array}{lll}\text { List Item } & 41.9 & 38.8 \\ \text { Critical Lure } & 52.4 & 53.9\end{array}$

$20 \mathrm{~ms}$

$\begin{array}{lll}\text { List Item } & 39.3 & 41.7 \\ \text { Critical Lure } & 50.8 & 51.6\end{array}$

Note. Presentation time was varied between subjects. 